\title{
350 及以上高牌号电工钢厚控的开发与应用
}

\author{
王天生
}

中冶南方（新余）冷轧新材料技术有限公司，江西 新余 338025

\begin{abstract}
[摘要] 首先 350 及以上牌号硅含量在 $2.7 \%$ 以上, 材质坚硬易脆断, 原料厚度波动大, 多为高频震荡的锯齿波。其次轧机设计 的最高牌号为 400 , 轧机牌坊的刚度不足, 辊缝跳动大。应用传统的前馈十反馈+秒流量的厚控方式轧制时, 成品厚度只能控 制在 $20 \mu$ 左右, 无法达到用户要求, 严重影响新产品开发及销售利润, 针对这一情况, 组织相关技术人员, 对 350 及以上牌 号进行厚控攻关。
\end{abstract}

[关键词]锯齿波; 反馈控制; 前馈控制

DOI：10.33142/aem.v3i3.3886 中图分类号: TM275 文献标识码：A

\section{Development and Application of Thickness Control of High Grade Electrical Steel of 350 and Above}

WANG Tiansheng

MCC Southern (Xinyu) Cold Rolling New Material Technology Co., Ltd., Xinyu, Jiangxi, 338025, China

\begin{abstract}
First of all, the silicon content of 350 and above grades is above 2.7\%, the material is hard and brittle, the thickness of the raw material fluctuates greatly, mostly high-frequency oscillating sawtooth waves. Secondly, the highest grade of the rolling mill design is 400 , the rigidity of the mill housing is insufficient and the roll gap is large. When rolling with the traditional thickness control method of feedforward + feedback + second flow rate, the thickness of the finished product can only be controlled at about $20 \mu$, which cannot meet user requirements and seriously affects new product development and sales profits. In view of this situation, organize relevant technical personnel to carry out thick control research on 350 and above brands.
\end{abstract}

Keywords: sawtooth wave; feedback control; feedforward control

\section{1 原因分析}

（1）热轧工序工艺不成熟，由于是新开发牌号，来料厚度成高频震荡锯齿波，对我厚控形成干扰;

(2) 前馈控制对来料要求相对较高, 对高频震荡波反应敏感, 反而加剧厚度波动。

(3) 350 及以上牌号含硅量较高硬度较大, 超出了原设计要求, 轧机本身刚性并不能完全满足其需求, 造成压下 精度损耗, 辊缝弹跳加剧, 致使最终成品厚度波动大。

\section{2 轧机厚控方式介绍}

单机架六辊轧机采用传统的前馈、反馈、秒流量的厚控方式组合的对产品进行控制。一、二道次由于轧制速度低 来料厚度波动大，采用前馈+反馈的方式进行轧制。三道次后采用三种控制方式共同作用的轧制方式。

\section{1 反馈控制}

反馈控制它通过出口带钢的厚度偏差值与目标厚度的比对, 使用积分控制器计算相应的修正值用于辊缝的补充。 反馈调节可有效消除热轧原料带来的大趋势的厚度波动, 使其整体厚度平缓。

\section{2 前馈控制}

前馈控制是用入口测厚仪在带钢未进入本机架之前测量出其入口厚度 $H_{i}$, 并与给定厚度值 $H_{0}$ 相比较, 当有厚度偏 差 $\Delta H$ 时, 便预先估计出可能产生的轧出厚度偏差 $\Delta h$, 从而确定为消除此 $\Delta h$ 值所需的辊缝调节量 $\Delta S$, 然后根据该检测 点进入本机架的时间和移动 $\Delta S$ 所需的时间, 提前对本机架进行厚度控制, 使得厚度的控制点正好就是 $\Delta H$ 的检测点。

\section{3 秒流量控制}

秒流量相等是秒流量控制的依据原理：入口厚度 $\times$ 入口速度 $=$ 出口厚度 $\times$ 出口速度，既 $v h_{0}=v h$ 。对带钢段 $h_{0}$ (入 口厚度）实测后通过延迟，当实测 $h_{0}$ 的带钢段进入变形区时根据此时实测的 $v$ (入口速度）及 $v$ （出口速度）即可精 确得到此带钢段的变形区出口厚度。中治新材六辊轧机的秒流量控制逻辑是根据激光测速仪的速度信号计算出初步的 轧后带钢厚度, 然后加上修正值 (可由测厚仪信号修正或通过经验公式计算) 得到经修正的计算厚差。根据这个计算 厚差, 转化为需调节的辊缝量, 使用比例积分控制器计算得到控制量, 经限幅后最终输出执行。 
在单独使用秒流量控制的时候发现了一种有趣的现象, 由图 1 可以看出, 在投入秒流量 AGC 控制时, 出入口测厚 仪所测得的纵向厚差曲线具有高度的相似性, 可以说是完全遗传了入口厚度曲线。此时的秒流量控制曲线无法改变带 钢的厚度曲线, 据此可认为秒流量的辊缝调节幅值偏小无法起到足够的作用。同时经过比对计算秒流量控制曲线的相 位角略微滞后于实际波形，因此需要将控制波形的相位整体提前。

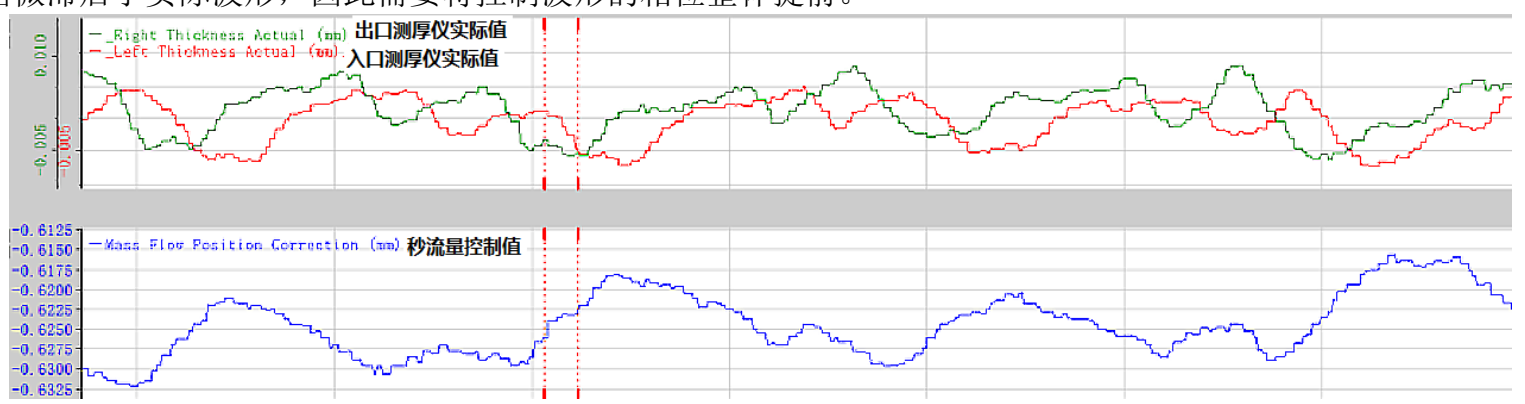

图 1 厚度曲线

\section{3 攻关方案及措施}

\section{1 取消前馈保留反馈控制}

前馈工作原理相对简单，其被用来补偿入口带钢厚度的不均匀性。它使用入口测厚仪来测量未轧制带钢的不规则 厚度偏差然后实施一个反作用于带钢传输给辊缝。所以在来料为高频震荡厚波的情况下, 前馈反而加剧出口厚度变化 量，不能起到消除厚差的作用，通过前一、二道次取消前馈，利用反馈控制的滞后性，消除高频锯齿波。

\section{2 增强秒流量控制量}

图 2 中 PIC 块为比例积分器, 其内部管脚如图所示, 当前管脚中 $\mathrm{IC}=0, \mathrm{EN}=1, \mathrm{~S}=0, \mathrm{HI}=1$, 根据 PIC 块真值表 可以知道, 目前比例积分器的并未发挥积分作用, 仅具有比例控制作用。W1 管脚输入为修正后的出口厚差值, 根据此 $\mathrm{PIC}$ 块作用知, 当前输出 $\mathrm{Y}=[(\mathrm{W} 1+\mathrm{W} 2)-(\mathrm{X} 1+\mathrm{X} 2)] * \mathrm{KP}, \mathrm{KP}=0.05, \mathrm{Y}$ 值为秒流量控制的未经补偿的液压缸动作修正值。所 以可以通过修改 KP 的设定值来达到改变幅值的目的。

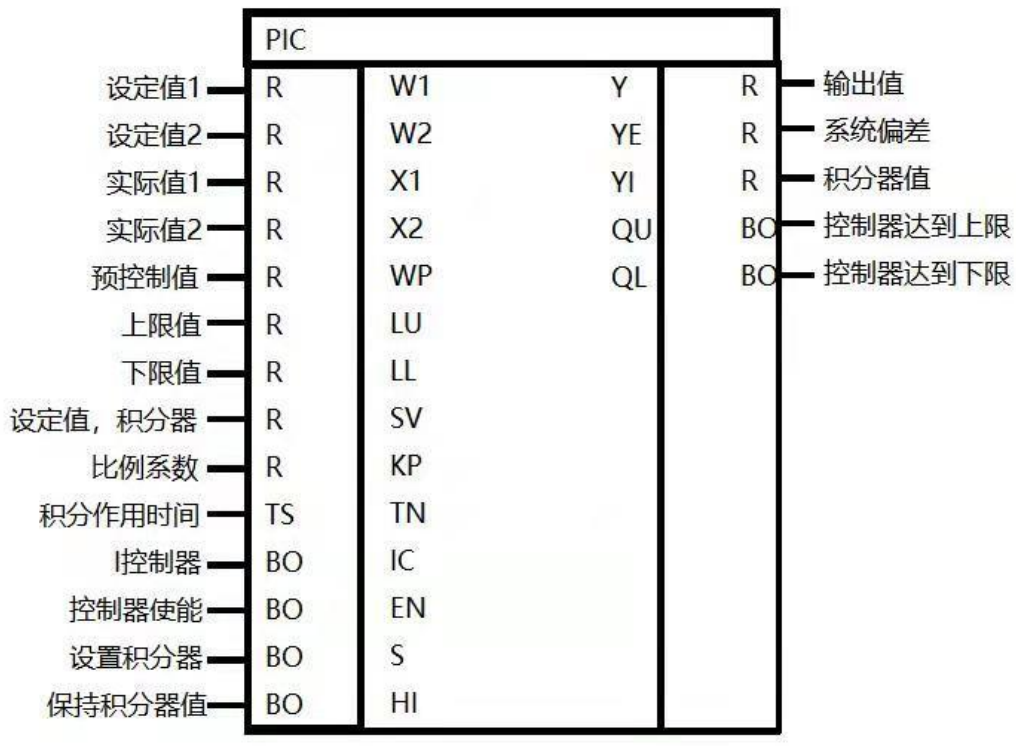

图 2 系统PIC 控制块

图 3 为模拟量队列块其管脚如图所示。XDH 管脚为入口测厚仪实际测量厚度, XV 管脚为入口段实际速度, $\mathrm{D}$ 管脚为 入口测厚仪至工作辊距离, TAD 管脚为队列输出延迟时间。当前秒流量的输出波形滞后, 可通过修改 TAD 管脚的值将入 口测厚仪队列输出延时减少。 


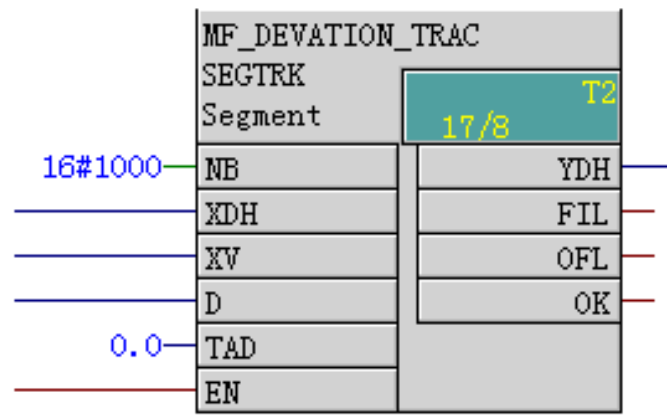

图 3 模拟量队列控制块

秒流量依据进出轧机金属体积相等原理进行厚度控制, 入口厚度 $\times$ 入口速度 $=$ 出口厚度 $\times$ 出口速度。所以只有在 高速稳定的情况下，秒流量作用更为明显，主要用于 3-6 道次厚度控制。

通过加大秒流量 Kp 时间减小 Ti 时间增加秒流量控制能力, 通过 L2 下发的牌号进行选择, 在车制高牌号时, 通过 位选块增加 $\mathrm{Kp}$ (管脚 $\mathrm{TAD}$ ) 时间为 0.5 , 非高牌号时为 0.2 。从而弥补没有前馈控制造成的压下量不足，并且实现更换牌 号自动选择参数的功能, 经过长期的跟踪实验出最合适的参数。

\section{4 结论}

(1) 攻关前后的厚度对比

攻关后从之前的 $\pm 8 \mu$ 减低到 $\pm 3 \mu$ 左右, 完全满足市场需求, 效果非常显著。

(2) 经济效益及应用前景

350 及 300 牌号从最初的实验牌号, 到现在年产近一万吨产量, 攻关后的厚控方式, 有效的保证了产品质量, 提升 其核心竞争力, 为公司创造巨大的经济效益, 也为更高牌号的新产品开发提供了有效的保证, 具有极大的应用前景。

\section{[参考文献]}

[1]胡少东.电磁搅拌对无取向电工钢 50W800 和 50W600 连铸板坏组织的影响 [D]. 江西: 江西理工大学, 2012.

[2] 徐星星, 秦镜, 赵海斌, 聂金成, 喻华宇. 新能源汽车用高牌号无取向电工钢的研究现状及发展趋势 [J]. 江西冶 金, 2020,40(3):6-11.

作者简介: 王天生（1987.5-), 长春大学, 电气工程及其自动化, 中冶南方（新余）冷轧新材料技术有限公司, 自动 化工程师。 